\title{
Finite-time synchronization of neural networks with discrete and distributed delays via periodically intermittent memory feedback control
}

\author{
Fei Yang ${ }^{1}$, Jun $\mathrm{Mei}^{2, *}$, Zhou $\mathrm{Wu}^{3}$ \\ ${ }^{1}$ Department of Automation, School of Power and Mechanical Engineering, Wuhan University, \\ Wuhan 430072, China \\ ${ }^{2}$ Department of Electrical, Electronic and Computer Engineering, University of Pretoria, Pretoria \\ 0028, South Africa \\ ${ }^{3}$ School of Automation, Chongqing University, Chongqing 400044, China \\ *meij0000@163.com
}

\begin{abstract}
In this paper, finite-time synchronization between two chaotic systems with discrete and distributed delays is investigated by using periodically intermittent memory feedback control. Based on finite-time stability theory, some novel and effective synchronization criteria of intermittent control are derived by means of linear matrix inequalities (LMIs) and differential inequality techniques. Furthermore, a necessary condition of finite-time synchronization of intermittent control is given for neural networks with discrete and distributed delays. A numerical example on two chaotic neural networks shows the effectiveness and correctness of the derived theoretical results. In addition, a secure communication synchronization problem is presented to demonstrate practical effectiveness of the proposed method.
\end{abstract}

\section{Introduction}

During the last two decades, chaos synchronization and control have been extensively investigated due to its importance in theory and its potential practical applications to various fields of mechanics, neural networks, biology and secure communication [1]-[4]. So far, many different notions of synchronization have been utilized, such as complete synchronization [5], lag synchronization [6], phase synchronization [7], projective synchronization [8], generalized synchronization [9]. Many effective control techniques including adaptive control [10], pinning control [11], impulsive control [12], feedback control [13, 14] and intermittent control [15]-[23] have been studied to drive system to achieve these synchronization problems. Among these control approaches, impulsive control and intermittent control as discontinuous control have become active research topics in engineering fields due to its practical application and easy implementation. The intermittent control, which is different from the impulsive control for activating only at some instant points, has a nonzero control horizon.

Recently, many important and interesting efforts have been made on stabilization and synchronization of nonlinear dynamical systems with or without time delays via the intermittent control [15]-[23]. However, to our best knowledge, most researches were mainly focused on asymptotical or exponential synchronization by using the intermittent control [15]-[23]. This means that the trajectories of drive system cannot track to the trajectories of response system within a finite time. 
However, in practical engineering fields, the systems might always be expected to be stabilized as quickly as possible even in finite time, namely, the so-called finite-time stability [24, 25]. For achieving finite-time stability, finite-time control techniques [26]-[31] are the effective and useful methods. Finite-time stability means the optimality in settling time. Furthermore, better robustness and disturbance rejection properties have been shown by using the finite-time control [32]. That is why finite-time control problem becomes a hot topic nowadays. Finite-time synchronization of systems without time delay by using a feedback controller was investigated $[13,30]$. Finite-time synchronization of systems with time delay by designing a non-smooth controller and a smooth controller were studied [33]-[35], [36]. The impulsive control method was designed for achieving finite-time synchronization of complex networks [19]. Finite-time synchronization of coupled neural networks is studied by using a discontinuous controller [31]. To the best of our knowledge, there are very few published papers considering finite-time synchronization of nonlinear dynamical systems via intermittent control [19, 20], [22]. Finite-time synchronization of complex networks by using periodically intermittent control is reported in $[19,20]$. In these papers, the time derivative of Lyapunov function $V(t)$ satisfied $\dot{V}(t) \leq 0$ when the network is without controller. However, for complex network, it is difficult to achieve this aim. Therefore, it is more significant to consider synchronization under $\dot{V}(t) \leq \beta V(t)(\beta>0)$. Though $\dot{V}(t) \leq \beta V(t)$ in non-control width was reported in [22], but it can be only achieved semi-global finite-time synchronization by using periodically intermittent control. Therefore it is very essential to investigate global finitetime synchronization by using periodically intermittent control with $\dot{V}(t) \leq \beta V(t)$ in non-control width. What's more, in [19, 20, 22], the authors have ignored an important condition that the first control width must less than the convergence time, otherwise, these studies were not a finite-time intermittent control problem. In this paper, we will solve these issues.

Motivated by the above issues, we study the finite-time synchronization of a general class of neural networks with discrete and distributed delays by means of periodically intermittent memory feedback control in this paper. Based on finite-time stability, some novel and useful synchronization criteria of intermittent memory control are developed by using the differential inequality methods and LMIs. The simulation results are given to demonstrate the effectiveness of the proposed approach. The proposed controller can be applied to practical secure communication with chaotic nodes, i.e., sender and receiver. The discrete and distributed time delays are complicated, which leads few possibility of interpreting the encrypted messages. The main contributions of this paper include: 1) the traditional assumptions on activation function have been released for satisfaction of Lipschitz condition; 2) the discrete and distributed delays are discussed; 3) a new smooth controller is designed to achieve finite-time synchronization via intermittent control; 4) a new finite-time synchronization using intermittent control is derived.

The paper is organized as follows. In Section 2, the synchronization problem to be studied is formulated, some useful lemmas and preliminaries are presented. In Section 3, some synchronization criteria for the neural networks with discrete and distributed delays are rigorously derived by using LMIs method. Numerical simulation is provided in Section 4. The proposed intermittent control scheme is applied to secure communication in Section 5, and conclusion is finally drawn in Section 6. 


\section{Preliminaries and Control Design}

\subsection{Problem Formulation}

Consider a class of neural networks with mixed time delays described by:

$$
\dot{x}(t)=-C x(t)+A g(x(t))+B g(x(t-\tau(t)))+D \int_{t-\sigma(t)}^{t} g(x(s)) \mathrm{d} s+J(t),
$$

where $x(t)=\left(x_{1}(t), x_{2}(t), \ldots, x_{n}(t)\right)^{T}$ is the vector of neuron states at time $t ; n$ represents the number of neurons in the network; $C=\operatorname{diag}\left(c_{1}, c_{2}, \ldots, c_{n}\right)$ is an $n \times n$ constant diagonal matrix with $c_{i}>0, i=1,2, \ldots, n ; A=\left(a_{i j}\right)_{n \times n}, B=\left(b_{i j}\right)_{n \times n}$ and $D=\left(d_{i j}\right)_{n \times n}$ are, respectively, the connection weight matrix, the discretely delayed connection weight matrix and the distributively delayed connection weight matrix; $g(x(t))=\left(g_{1}\left(x_{1}(t)\right), g_{2}\left(x_{2}(t)\right), \ldots, g_{n}\left(x_{n}(t)\right)\right)^{T}$ is a diagonal mapping where $g_{i}(\cdot), i=1,2, \ldots, n$, represents the neuron activation function; $J(t)=\left(J_{1}(t), J_{2}(t), \ldots, J_{n}(t)\right)^{T}$ is an external input vector; $\tau(t)$ and $\sigma(t)$ are the discrete delay and the distributed delay, respectively.

In this paper, we refer to model (1) as the drive system, the corresponding response system is designed as

$$
\dot{y}(t)=-C y(t)+A g(y(t))+B g(y(t-\tau(t)))+D \int_{t-\sigma(t)}^{t} g(y(s)) \mathrm{d} s+J(t)+u(t),
$$

where $y(t)$ is the state vector of the response system (2), the system matrices $C, A, B$ and $D$ have the same definitions as in (1), and $u(t)$ is the appropriate control input that will be designed in order to obtain a certain control objective.

By defining the error system as $e(t)=y(t)-x(t)$, yields the synchronization error dynamical system described by

$$
\dot{e}(t)=-C e(t)+A f(e(t))+B f(e(t-\tau(t)))+D \int_{t-\sigma(t)}^{t} f(e(s)) \mathrm{d} s+u(t),
$$

where $f(e(t)):=g(y(t))-g(x(t))$.

\subsection{Some Assumptions and Definition}

Assumption 1. ([37]): Each activation function $f_{i}(\cdot)$ in (3) satisfied $f(0)=0$ is continuous and bounded, and there exist constants $l_{i}^{-}$and $l_{i}^{+}$such that

$$
l_{i}^{-} \leq \frac{f_{i}\left(s_{1}\right)-f_{i}\left(s_{2}\right)}{s_{1}-s_{2}} \leq l_{i}^{+}, \quad i=1,2, \ldots, n,
$$

where $s_{1}, s_{2} \in R$, and $s_{1} \neq s_{2}$.

Assumption 2. The discrete delay $\tau(t)$ and the distributed delay $\sigma(t)$ satisfy

$$
0 \leq \tau(t) \leq \tau, \dot{\tau}(t) \leq \tau, 0 \leq \sigma(t) \leq \sigma
$$


Assumption 3. ([29]): Let $0<\rho<1$ and $\lambda>0$, then there exists a condition function $h:[0, \infty) \rightarrow[0, \infty)$ with $h(0) \geq 0$, such that the following inequality holds:

$$
h(t)-h(t+\xi) \leq-\lambda \int_{t+\xi}^{t} h^{\rho}(s) \mathrm{d} s,
$$

for any $-d \leq \xi \leq 0$ and $d>0$.

Definition 1. (Finite-time synchronization) The systems (1) and (2) are said to achieve local synchronization in finite-time $t^{*}$ if there exists a positive constant $\kappa$ such that for any solution of systems (1) and (2) with different initial values $\phi, \varphi \in \Omega=\left\{\psi \in \mathcal{C}\left(\left[t_{0}-\tau, t_{0}\right], R^{n}\right),\|\psi\|<\kappa\right\}$, and $t^{*}$ depends on the initial state vector values $\phi$ and $\varphi$, for any $t \geq t^{*}$, such that

$$
\left\|y_{i}(t)-x_{i}(t)\right\|=0, \text { as } t \rightarrow t^{*}
$$

hold for any $i=1,2, \ldots, n$, where $x(t)=\left(x_{1}(t), \ldots, x_{n}(t)\right)^{T} \in R^{n}$ and $y(t)=\left(y_{1}(t), \ldots, y_{n}(t)\right.$ )$^{T} \in R^{n}$. Furthermore, if $\Omega=\mathcal{C}\left([-\tau, 0], R^{n}\right)$, the systems (1) and (2) are said to achieve global synchronization in finite-time $t^{*}$.

\subsection{Some Useful Lemmas and Proposition}

Lemma 1. Assume that a continuous, positive-definite function $V(t)$ defined on a neighborhood $u \in R^{n}$ of the origin, and satisfies the following differential inequality:

$$
\dot{V}(t) \leq-\alpha V^{\eta}(t)-p V(t), \quad \forall t \in u \backslash\{0\},
$$

where $\alpha>0,0<\eta<1, p>0$ are three constants. Then, for any given $t_{0}, V(t)$ satisfies the following inequality:

$$
\begin{array}{cc}
V^{1-\eta}(t) \exp \{(1-\eta) p t\} \leq & V^{1-\eta}\left(t_{0}\right) \exp \left\{(1-\eta) p t_{0}\right\}+\frac{\alpha}{p}\left[\exp \left\{(1-\eta) p t_{0}\right\}-\right. \\
\exp \{(1-\eta) p t\}], & t_{0} \leq t \leq t_{1},
\end{array}
$$

and

$$
V(t) \equiv 0, \quad \forall t \geq t_{1}
$$

with $t_{1}$ given by

$$
t_{1} \leq \frac{\ln \left(1+\frac{p}{\alpha} V^{1-\eta}(0)\right)}{p(1-\eta)},
$$

for $t_{0}=0$.

Proof: Consider the following differential equation:

$$
\dot{X}(t)=-\alpha X^{\eta}(t)-p X(t), \quad X\left(t_{0}\right)=V\left(t_{0}\right) .
$$

By multiplying $\exp \{p t\}$, we have

$$
\frac{d(\exp \{p t\} X(t))}{d t}=-\alpha(\exp \{p t\} X(t))^{\eta} \exp \{(1-\eta) p t\} .
$$


Although this differential equation does not satisfy the global Lipschitz condition, the unique solution to this equation can be found as

$$
\begin{aligned}
X^{1-\eta}(t) \exp \{(1-\eta) p t\}= & X^{1-\eta}\left(t_{0}\right) \exp \left\{(1-\eta) p t_{0}\right\}+\frac{\alpha}{p}\left[\exp \left\{(1-\eta) p t_{0}\right\}-\right. \\
& \exp \{(1-\eta) p t\}], \quad t_{0}<t \leq t_{1},
\end{aligned}
$$

and $X(t) \equiv 0, \quad \forall t \geq t_{1}$.

It is direct to prove that $x(t)$ is differential for $t>t_{0}$. From the comparison principle, one obtains

$$
\begin{aligned}
& V^{1-\eta}(t) \exp \{(1-\eta) p t\} \leq V^{1-\eta}\left(t_{0}\right) \exp \left\{(1-\eta) p t_{0}\right\}+\frac{\alpha}{p}\left[\exp \left\{(1-\eta) p t_{0}\right\}-\right. \\
& \exp \{(1-\eta) p t\}], \quad t_{0}<t \leq t_{1},
\end{aligned}
$$

and $V(t) \equiv 0, \quad \forall t \geq t_{1}$.

Then $t_{1}$ follows the formulary (6) with $t_{0}=0$.

Lemma 2. ([38]): If $a_{1}, a_{2}, \ldots, a_{n} \geq 0$ and $0<q<1$, then

$$
\left(\sum_{i=1}^{n} a_{i}\right)^{q} \leq \sum_{i=1}^{n} a_{i}^{q}
$$

Lemma 3. ([39]): For any positive definite matrix $M>0$, scalars $\gamma_{2}>\gamma_{1}>0$ and vector function $\omega:\left[\gamma_{1}, \gamma_{2}\right]$ such that the integrations concerned are well defined, then the following inequality holds:

$$
\left(\int_{\gamma_{1}}^{\gamma_{2}} \omega(s) \mathrm{d} s\right)^{T} M\left(\int_{\gamma_{1}}^{\gamma_{2}} \omega(s) \mathrm{d} s\right) \leq\left(\gamma_{2}-\gamma_{1}\right) \int_{\gamma_{1}}^{\gamma_{2}} \omega^{T}(s) M \omega(s) \mathrm{d} s .
$$

\subsection{Design a finite-time controller}

In this subsection, a new intermittent feedback controller (i.e., periodically intermittent memory feedback control) is designed for achieving finite-time synchronization described by

$$
\left\{\begin{aligned}
u(t)= & -k_{1}(y(t)-x(t))-k_{2} \frac{\left(\lambda_{\max }(P)\right)^{\frac{1+\mu}{2}}}{\lambda_{\min }(P)} \operatorname{sign}(e(t))|e(t)|^{\mu}-k_{3} \int_{t-\sigma(t)}^{t} f(e(s)) \mathrm{d} s \\
& l T \leq t<l T+\delta \\
u(t)=0, & l T+\delta \leq t<(l+1) T
\end{aligned}\right.
$$

where $|e(t)|^{\mu}=\left(\left|e_{1}(t)\right|^{\mu}, \ldots,\left|e_{n}(t)\right|^{\mu}\right)^{T}$ and $\operatorname{sign}(e(t))=\operatorname{diag}\left(\operatorname{sign}\left(e_{1}(t)\right), \ldots, \operatorname{sign}\left(e_{n}(t)\right)\right)^{T}$; constants $k_{1}, k_{2}, k_{3}$ are gain coefficients to be determined; the real number $\mu$ satisfies $0<\mu<1$. Denote $\lambda_{\max }(P)$ and $\lambda_{\min }(P)$ as the maximum and minimum eigenvalue of the symmetric positive definite matrix $P$, respectively. $T>0$ is the control period, $T>\delta>0$ is called the control width (control duration), and $\theta=\delta / T$ is called the rate of control duration. $\ell=\{1,2, \ldots, \iota\}$ is a finite natural number set and $l \in \ell$.

By substituting (7) into (3), we obtain

$$
\left\{\begin{aligned}
& \dot{e}(t)=-C e(t)+A f(e(t))+B f(e(t-\tau(t)))+D \int_{t-\sigma(t)}^{t} f(e(s)) \mathrm{d} s-k_{1} e(t)- \\
& k_{2} \frac{\left(\lambda_{\max }(P)\right)^{\frac{1+\mu}{2}}}{\lambda_{\min }(P)} \operatorname{sign}(e(t))|e(t)|^{\mu}-k_{3} \int_{t-\sigma(t)}^{t} f(e(s)) \mathrm{d} s, l T \leq t<l T+\theta T \\
& \dot{e}(t)=-C e(t)+A f(e(t))+B f(e(t-\tau(t)))+D \int_{t-\sigma(t)}^{t} f(e(s)) \mathrm{d} s \\
& l T+\theta T \leq t<(l+1) T .
\end{aligned}\right.
$$




\section{Criteria for synchronization}

In order to derive the finite-time synchronization criteria for the two chaotic systems (1) and (2), we firstly give the following lemmas that will play an important role in the proof of the main results.

Lemma 4. Suppose that function $V(t)$ is continuous and non-negative when $t \in[0, \infty)$ and satisfies the following conditions:

$$
\left\{\begin{array}{l}
\dot{V}(t) \leq-\alpha V^{\eta}(t)-p_{1} V(t), \quad l T \leq t<l T+\theta T \\
\dot{V}(t) \leq p_{2} V(t), \quad l T+\theta T \leq t<(l+1) T
\end{array}\right.
$$

where $\alpha, T, p_{1}, p_{2}>0,0<\theta, \eta<1$, are any constants and $l \in \ell$. If

$$
\theta p_{1}-(1-\theta) p_{2}>0
$$

then the following inequality holds:

$$
\begin{aligned}
V^{1-\eta}(t) \exp \left\{(1-\eta) p_{1} t\right\} \leq & \exp \left\{(1-\eta)\left(p_{1}+p_{2}\right)(1-\theta) t\right\}\left[V^{1-\eta}(0)-\frac{\alpha}{p_{1}}(\exp \{(1\right. \\
& \left.\left.\left.-\eta) p_{1} \theta t\right\} \exp \left\{-(1-\eta) p_{2}(1-\theta) t\right\}-1\right)\right], \quad t \geq 0
\end{aligned}
$$

The proof is given in Appendix.

In the following, we will derive some sufficient finite-time synchronization criteria for the drive system (1) and response system (2) based on periodically intermittent memory feedback control and differential inequality techniques. To present the main results of this section, we first denote

$$
\begin{aligned}
L_{1}: & =\operatorname{diag}\left(l_{1}^{+} l_{1}^{-}, \ldots, l_{n}^{+} l_{n}^{-}\right) \\
L_{2} & :=\operatorname{diag}\left(\frac{l_{1}^{+}+l_{1}^{-}}{2}, \ldots, \frac{l_{n}^{+}+l_{n}^{-}}{2}\right) \\
L_{3} & :=\operatorname{diag}\left(l_{1}^{+} l_{1}^{+}, \ldots, l_{n}^{+} l_{n}^{+}\right) \\
L_{4} & :=\operatorname{diag}\left(l_{1}^{-} l_{1}^{-}, \ldots, l_{n}^{-} l_{n}^{-}\right) \\
L_{5} & :=\operatorname{diag}\left(l_{1}^{-}, \ldots, l_{n}^{-}\right) \\
L_{6} & :=\operatorname{diag}\left(l_{1}^{+}, \ldots, l_{n}^{+}\right)
\end{aligned}
$$

Theorem 1. Assume that Assumptions 1-3 hold. For given any positive constants $\alpha$, $\beta$, if there exist four symmetric positive definite matrices $P_{i}(i=1,2,3,4)$, and four diagonal positive definite matrices $P_{5}=\operatorname{diag}\left(p_{51}, \ldots, p_{5 n}\right), P_{6}=\operatorname{diag}\left(p_{61}, \ldots, p_{6 n}\right), \wedge=\operatorname{diag}\left(\pi_{1}, \ldots, \pi_{n}\right)$ and $\Gamma=\operatorname{diag}\left(\xi_{1}, \ldots, \xi_{n}\right)$ such that the following conditions hold

$$
\Omega=\left[\begin{array}{ccccc}
\Omega_{11} & 0 & \Omega_{13} & P_{1} B & P_{1} E-k_{3} P_{1} \\
* & \Omega_{22} & 0 & L_{2} \Gamma & 0 \\
* & * & \Omega_{33} & 0 & 0 \\
* & * & * & \Omega_{44} & 0 \\
* & * & * & * & -\frac{\exp \{-\alpha \tau\} P_{4}-\frac{2 k_{2}}{\lambda} P_{4}}{\sigma}
\end{array}\right]<0
$$




$$
\begin{gathered}
\Phi=\left[\begin{array}{ccccc}
\Phi_{11} & 0 & \Phi_{13} & P_{1} B & P_{1} E \\
* & \Phi_{22} & 0 & L_{2} \Gamma & 0 \\
* & * & \Phi_{33} & 0 & 0 \\
* & * & * & -\Gamma & 0 \\
* & * & * & * & -\frac{\exp \{-\alpha \tau\} P_{4}}{\sigma}
\end{array}\right]<0, \\
\theta \alpha-(1-\theta) \beta>0, \\
\theta<\min \left\{1, \frac{\beta}{\alpha+\beta}+\sqrt{\left.\frac{\ln \left(1+\frac{\alpha V^{\frac{1-\mu}{2}}}{2 k_{2}}\right)}{\frac{1-\mu}{2} T(\alpha+\beta)}+\left(\frac{\beta}{2(\alpha+\beta)}\right)^{2}\right\}},\right.
\end{gathered}
$$

where

$$
\begin{aligned}
& \Omega_{11}=-P_{1} C-C^{T} P_{1}+\alpha P_{1}-2 k_{1} P_{1}+P_{2}+L_{5} P_{5} L_{5}+L_{6} P_{6} L_{6}-\frac{2 k_{2}}{\lambda} P_{2}-\frac{2 k_{2}}{\lambda} L_{5} P_{5} L_{5}- \\
& \frac{2 k_{2}}{\lambda} L_{6} P_{6} L_{6}-L_{1} \wedge, \Omega_{13}=-P_{5} L_{5}-P_{6} L_{6}+P_{1} A+\frac{2 k_{2}}{\lambda} P_{5} L_{5}+\frac{2 k_{2}}{\lambda} P_{6} L_{6}+L_{2} \wedge, \\
& \Omega_{22}=-\exp \{-\alpha \tau\}(1-\tau) P_{2}+\frac{2 k_{2}}{\lambda} P_{2}-L_{1} \Gamma, \Omega_{33}=P_{3}+\sigma P_{4}+P_{5}+P_{6}-\frac{2 k_{2}}{\lambda} P_{3}- \\
& \frac{2 k_{2}}{\lambda} P_{5}-\frac{2 k_{2}}{\lambda} P_{6}-\frac{2 k_{2}}{\lambda} \sigma P_{4}-\wedge, \Omega_{44}=-\exp \{-\alpha \tau\}(1-\tau) P_{3}+\frac{2 k_{2}}{\lambda} P_{3}-\Gamma, \\
& \Phi_{11}=-P_{1} C-C^{T} P_{1}-\beta P_{1}+P_{2}+L_{5} P_{5} L_{5}+L_{6} P_{6} L_{6}-L_{1} \wedge, \Phi_{13}=-P_{5} L_{5}-P_{6} L_{6}+ \\
& P_{1} A+L_{2} \wedge, \Phi_{22}=-\exp \{-\alpha \tau\}(1-\tau) P_{2}-L_{1} \Gamma, \Phi_{33}=P_{3}+\sigma P_{4}+P_{5}+P_{6}-\wedge, \\
& \Phi_{44}=-\exp \{-\alpha \tau\}(1-\tau) P_{3}-\Gamma,
\end{aligned}
$$

then the error system (8) is synchronized under the periodically intermittent memory controller (7) in finite time:

$$
T_{1}=\frac{\ln \left(1+\frac{\alpha V^{\frac{1-\mu}{2}}(0)}{2 k_{2}}\right)}{\frac{1-\mu}{2}(\theta \alpha-(1-\theta) \beta)} .
$$

Proof: Consider the following Lyapunov function for the error system (8)

$$
V(t)=\sum_{i=1}^{6} V_{i}(t)
$$

where

$$
V_{1}(t)=e^{T}(t) P_{1} e(t)
$$




$$
\begin{gathered}
V_{2}(t)=\int_{t-\tau(t)}^{t} \exp \{\alpha(s-t)\} e^{T}(s) P_{2} e(s) \mathrm{d} s \\
V_{3}(t)=\int_{t-\tau(t)}^{t} \exp \{\alpha(s-t)\} f^{T}(e(s)) P_{3} f(e(s)) \mathrm{d} s, \\
V_{4}(t)=\int_{-\sigma}^{0} \int_{t+\xi}^{t} \exp \{\alpha(s-t)\} f^{T}(e(s)) P_{4} f(e(s)) \mathrm{d} s \mathrm{~d} \xi \\
V_{5}(t)=\int_{0}^{t} \exp \{\alpha(s-t)\} \sum_{i=1}^{n} p_{5 i}\left(f_{i}\left(e_{i}(s)\right)-l_{i}^{-} e_{i}(s)\right)^{T}\left(f_{i}\left(e_{i}(s)\right)-l_{i}^{-} e_{i}(s)\right) \mathrm{d} s, \\
V_{6}(t)=\int_{0}^{t} \exp \{\alpha(s-t)\} \sum_{i=1}^{n} p_{6 i}\left(l_{i}^{+} e_{i}(s)-f_{i}\left(e_{i}(s)\right)\right)^{T}\left(l_{i}^{+} e_{i}(s)-f_{i}\left(e_{i}(s)\right)\right) \mathrm{d} s .
\end{gathered}
$$

Referring Assumptions 2 and Lemma 3, we can calculate the time derivative of $V_{i}(t)(i=1,2, \ldots$, 6) as

$$
\begin{gathered}
\dot{V}_{1}(t)=-\alpha V_{1}(t)+\alpha e^{T}(t) P_{1} e(t)+2 e^{T}(t) P_{1} \dot{e}(t) \\
\dot{V}_{2}(t)=-\alpha V_{2}(t)+e^{T}(t) P_{2} e(t)-\exp \{-\alpha \tau(t)\}(1-\dot{\tau}(t)) e^{T}(t-\tau(t)) P_{2} e(t-\tau(t)) \\
\leq-\alpha V_{2}(t)+e^{T}(t) P_{2} e(t)-\exp \{-\alpha \tau\}(1-\tau) e^{T}(t-\tau(t)) P_{2} e(t-\tau(t)), \\
\dot{V}_{3}(t)=-\alpha V_{3}(t)+f^{T}(e(t)) P_{3} f(e(t))-\exp \{-\alpha \tau(t)\}(1-\dot{\tau}(t)) f^{T}(e(t-\tau(t))) P_{3} \\
\quad f(e(t-\tau(t))) \\
\leq-\alpha V_{3}(t)+f^{T}(e(t)) P_{3} f(e(t))-\exp \{-\alpha \tau\}(1-\tau) f^{T}(e(t-\tau(t))) P_{3} f(e(t-\tau(t))), \\
\dot{V}_{4}(t)=-\alpha V_{4}(t)+\sigma f^{T}(e(t)) P_{4} f(e(t))-\int_{-\sigma}^{0} \exp \{\alpha \xi\} f^{T}(e(t+\xi)) P_{4} f(e(t+\xi)) \mathrm{d} \xi \\
\leq-\alpha V_{4}(t)+\sigma f^{T}(e(t)) P_{4} f(e(t))-\frac{\exp \{-\alpha \sigma\}}{\sigma}\left(\int_{t-\sigma(t)}^{t} f(e(s)) \mathrm{d} s\right)^{T} P_{4} \\
\quad\left(\int_{t-\sigma(t)}^{t} f(e(s) \mathrm{d} s),\right. \\
\dot{V}_{5}(t)=-\alpha V_{5}(t)+f^{T}(e(t)) P_{5} f(e(t))-2 f^{T}(e(t)) P_{5} L_{5} e(t)+e^{T}(t) L_{5} P_{5} L_{5} e(t)
\end{gathered}
$$




$$
\dot{V}_{6}(t)=-\alpha V_{6}(t)+f^{T}(e(t)) P_{6} f(e(t))-2 e^{T}(t) P_{6} L_{6} f(e(t))+e^{T}(t) L_{6} P_{6} L_{6} e(t) .
$$

From $0<\mu<1$ and Lemma 2, we have

$$
\sum_{i=1}^{n}\left|e_{i}(t)\right|^{1+\mu}=\sum_{i=1}^{n}\left(e_{i}^{T}(t) e_{i}(t)\right)^{\frac{1+\mu}{2}} \geq\left(\sum_{i=1}^{n} e_{i}^{T}(t) e_{i}(t)\right)^{\frac{1+\mu}{2}} .
$$

Let $\lambda>0$, then from Assumption 3 and Lemma 2 we have

$$
\begin{gathered}
\frac{2 k_{2}}{\lambda}\left[e^{T}(t) P_{2} e(t)-e^{T}(t-\tau(t)) P_{2} e(t-\tau(t))\right] \leq-2 k_{2} \int_{t-\tau}^{t}\left(e^{T}(s) P_{2} e(s)\right)^{\frac{1+\mu}{2}} \mathrm{~d} s \\
\leq-2 k_{2}\left(\int_{t-\tau}^{t} e^{T}(s) P_{2} e(s) \mathrm{d} s\right)^{\frac{1+\mu}{2}} \\
\frac{2 k_{2}}{\lambda}\left[f^{T}(e(t)) P_{3} f(e(t))-f^{T}(e(t-\tau(t))) P_{3} f(e(t-\tau(t)))\right] \\
\leq-2 k_{2}\left(\int_{t-\tau(t)}^{t} \exp \{\alpha(s-t)\} f^{T}(e(s)) P_{3} f(e(s)) \mathrm{d} s\right)^{\frac{1+\mu}{2}} .
\end{gathered}
$$

Let $\lambda>0$, then by Assumption 3 and Lemma 2, we get

$$
\begin{aligned}
& \frac{2 k_{2}}{\lambda}\left[\sigma f^{T}(e(t)) P_{4} f(e(t))-\int_{t-\sigma}^{t} f^{T}(e(s)) P_{4} f(e(s)) \mathrm{d} s\right] \\
\leq & -2 k_{2}\left(\int_{-\sigma}^{0} \int_{t+\xi}^{t} \exp \{\alpha(s-t)\} f^{T}(e(\xi)) P_{4} f(e(\xi)) \mathrm{d} \xi \mathrm{d} s\right)^{\frac{1+\mu}{2}} .
\end{aligned}
$$

From Assumption 3, we can get

$$
\begin{aligned}
& \frac{2 k_{2}}{\lambda}\left[\sum_{i=1}^{n} p_{5 i}\left(f_{i}\left(e_{i}(t)\right)-l_{i}^{-} e_{i}(t)\right)^{T}\left(f_{i}\left(e_{i}(t)\right)-l_{i}^{-} e_{i}(t)\right)\right] \\
\leq & -2 k_{2}\left[\int_{0}^{t} \exp \{\alpha(s-t)\} \sum_{i=1}^{n} p_{5 i}\left(f_{i}\left(e_{i}(s)\right)-l_{i}^{-} e_{i}(s)\right)^{T}\left(f_{i}\left(e_{i}(s)\right)-l_{i}^{-} e_{i}(s)\right) \mathrm{d} s\right]^{\frac{1+\mu}{2}}, \\
& \frac{2 k_{2}}{\lambda}\left[\sum_{i=1}^{n} p_{6 i}\left(l_{i}^{+} e_{i}(t)-f_{i}\left(e_{i}(t)\right)\right)^{T}\left(l_{i}^{+} e_{i}(t)-f_{i}\left(e_{i}(t)\right)\right)\right] \\
\leq & -2 k_{2}\left[\int_{0}^{t} \exp \{\alpha(s-t)\} \sum_{i=1}^{n} p_{6 i}\left(l_{i}^{+} e_{i}(s)-f_{i}\left(e_{i}(s)\right)\right)^{T}\left(l_{i}^{+} e_{i}(s)-f_{i}\left(e_{i}(s)\right)\right) \mathrm{d} s\right]^{\frac{1+\mu}{2}},
\end{aligned}
$$

By Assumption 1, we have

$$
\begin{aligned}
& \left(f_{i}\left(e_{i}(t)\right)-l_{i}^{+} e_{i}(t)\right)\left(f_{i}\left(e_{i}(t)\right)-l_{i}^{-} e_{i}(t)\right) \leq 0 \\
& \left(f_{i}\left(e_{i}(t-\tau(t))\right)-l_{i}^{+} e_{i}(t-\tau(t))\right)\left(f_{i}\left(e_{i}(t-\tau(t))\right)-l_{i}^{-} e_{i}(t-\tau(t))\right) \leq 0
\end{aligned}
$$


for $i=1,2, \ldots, n$.

Then

$$
\begin{gathered}
\sum_{i=1}^{n} \pi_{i}\left[\begin{array}{c}
e(t) \\
f(e(t))
\end{array}\right]^{T}\left[\begin{array}{cc}
l_{i}^{+} l_{i}^{-} \varepsilon_{i} \varepsilon_{i}^{T} & -\frac{l_{i}^{+}+l_{i}^{-}}{2} \varepsilon_{i} \varepsilon_{i}^{T} \\
-\frac{l_{i}^{+}+l_{i}^{-}}{2} \varepsilon_{i} \varepsilon_{i}^{T} & \varepsilon_{i} \varepsilon_{i}^{T}
\end{array}\right]\left[\begin{array}{c}
e(t) \\
f(e(t))
\end{array}\right] \leq 0, \\
\sum_{i=1}^{n} \xi_{i}\left[\begin{array}{c}
e(t-\tau(t)) \\
f(e(t-\tau(t)))
\end{array}\right]^{T}\left[\begin{array}{cc}
l_{i}^{+} l_{i}^{-} \varepsilon_{i} \varepsilon_{i}^{T} & -\frac{l_{i}^{+}+l_{i}^{-}}{2} \varepsilon_{i} \varepsilon_{i}^{T} \\
-\frac{l_{i}^{+}+l_{i}^{-}}{2} \varepsilon_{i} \varepsilon_{i}^{T} & \varepsilon_{i} \varepsilon_{i}^{T}
\end{array}\right]\left[\begin{array}{c}
e(t-\tau(t)) \\
f(e(t-\tau(t)))
\end{array}\right] \leq 0
\end{gathered}
$$

for $i=1,2, \ldots, n$.

Where $\varepsilon_{i}$ denotes the unit column vector having a "1" element on its $i$ th row and zeros elsewhere.

When $l T \leq t<(l+\theta) T$, for $l \in \ell$, it follows from inequalities (18)-(31) and error system (8) that

$$
\dot{V}(t) \leq \eta^{T}(t) \Omega \eta(t)-2 k_{1} V^{\frac{1+\mu}{2}}(t)-\alpha V(t)
$$

where

$$
\eta(t)=\left[e^{T}(t) e^{T}(t-\tau(t)) f^{T}(t) f^{T}(t-\tau(t))\left(\int_{t-\sigma(t)}^{t} f(e(s)) \mathrm{d} s\right)^{T}\right]^{T},
$$

Then by (12), $\dot{V}(t)$ becomes

$$
\dot{V}(t) \leq-2 k_{2} V^{\frac{1+\mu}{2}}(t)-\alpha V(t) .
$$

When $(l+\theta) T \leq t<(l+1) T$, for $l \in \ell$, it follows from (8), (18)-(31) and (13) that

$$
\begin{aligned}
V(t) & \leq-\alpha V(t)+\eta^{T}(t) \Phi \eta(t)+(\alpha+\beta) V_{1}(t) \\
& \leq-\alpha V(t)+\eta^{T}(t) \Phi \eta(t)+(\alpha+\beta) V(t) \\
& \leq \beta V(t) .
\end{aligned}
$$

Namely, from (32) and (33), we have

$$
\left\{\begin{array}{l}
\dot{V}(t) \leq-2 k_{2} V^{\frac{1+\mu}{2}}(t)-\alpha V(t), l T \leq t<l T+\theta T \\
\dot{V}(t) \leq \beta V(t), \quad l T+\theta T \leq t<(l+1) T
\end{array}\right.
$$

By Lemma 4, we have

$$
\begin{aligned}
V^{\frac{1-\mu}{2}}(t) \exp \left\{\frac{1-\mu}{2} \alpha t\right\} \leq & \exp \left\{\frac{1-\mu}{2}(\alpha+\beta)(1-\theta) t\right\}\left[V^{1-\eta}(0)-\right. \\
& \left.\frac{2 k_{2}}{\alpha}\left(\exp \left\{\frac{1-\mu}{2}(\alpha \theta-\beta(1-\theta)) t\right\}-1\right)\right], \quad t \geq 0
\end{aligned}
$$

According to Lemma 1, we obtain

$$
t \leq \frac{\ln \left(1+\frac{\alpha V^{\frac{1-\mu}{2}}(0)}{2 k_{2}}\right)}{\frac{1-\mu}{2}(\alpha \theta-\beta(1-\theta))}=T_{1} .
$$

Thus, the finite-time synchronization between the drive system (1) and the response system (2) is achieved with the convergence time in (36). This completed the proof. 
Remark 1. In order to achieve finite-time synchronization via periodically intermittent memory

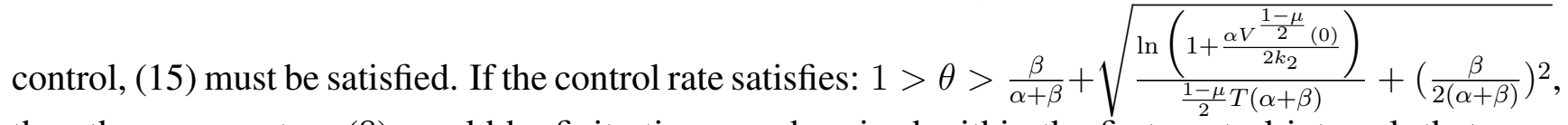
then the error system (8) would be finite-time synchronized within the first control interval, that is, the case is the continuous control problem. If $\theta=1$, the error system (8) is synchronized by the continuous control. However, this trivial case will not be discussed in this paper, as it has been considered in previous works. If $\theta<\min \left\{1, \frac{\beta}{\alpha+\beta}+\sqrt{\frac{\ln \left(1+\frac{\alpha V^{\frac{1-\mu}{2}}}{2 k_{2}}(0)\right.}{\frac{1-\mu}{2} T(\alpha+\beta)}+\left(\frac{\beta}{2(\alpha+\beta)}\right)^{2}}\right\}$, the error system (8) achieves finite-time synchronization via intermittent control, this restrict condition is essential but ignored in our previous paper [20].

Remark 2. From (36), we can easily see that the control rate $\theta$, the gain coefficient $k_{2}$, the index constant $\mu$ and the scalars $\alpha, \beta$ have an important role in finite-timely synchronizing the error system (8), and the parameters $\theta, k_{2}, \mu, \alpha, \beta$ are the decision variables of the convergence time. The role of the parameters $\mu, k_{2}$ in convergence time have been discussed by Ref. [30], the convergence time which is determined by the parameter $\theta$ has been studied by Refs. [19, 20]. In this paper, we will focus on the additional decision variables $\alpha, \beta$ influence the convergence time (36). Denotes $T(\alpha, \beta)=\frac{\ln \left(1+\frac{\alpha V \frac{1-\mu}{2}}{2 k_{2}}(0)\right.}{\frac{1-\mu}{2}(\alpha \theta-\beta(1-\theta))}$, it yields $\frac{\partial T}{\partial \alpha}<0$, we follow that $T(\alpha, \beta)$ is the strictly monotone decreasing function for the variable $\alpha$, and we obtain that the function $T(\alpha, \beta)$ is the strictly monotone increasing function with the variable $\beta$. Hence, the larger the parameter $\alpha$ is, the faster the convergence time will be; the larger the parameter $\beta$ is, the slower the convergence time will be.

Suppose $\beta=0$, based on Theorem 1, the following Theorem 2 is obtained.

Theorem 2. Assume that Assumptions 1-3 hold. For given any positive constant $\alpha$, if there exist four symmetric positive definite matrices $P_{i}(i=1,2,3,4)$, and four diagonal positive definite matrices $P_{5}=\operatorname{diag}\left(p_{51}, \ldots, p_{5 n}\right), P_{6}=\operatorname{diag}\left(p_{61}, \ldots, p_{6 n}\right), \wedge=\operatorname{diag}\left(\pi_{1}, \ldots, \pi_{n}\right)$ and $\Gamma=\operatorname{diag}\left(\xi_{1}, \ldots, \xi_{n}\right)$ such that (12), (13) $\left.\right|_{\beta=0}$, and (15) $\left.\right|_{\beta=0}$ hold, then the error system (8) is synchronized under the periodically intermittent memory controller (7) in finite time:

$$
T_{2}=\frac{\ln \left(1+\frac{\alpha V^{\frac{1-\mu}{2}}(0)}{2 k_{2}}\right)}{\frac{1-\mu}{2} \theta \alpha} .
$$

Proof: With the help of Lemma 2 and Lemma 5 under $p_{2}=0$, we can easily derive this theorem.

For a special case when the control rate $\theta=1$, and $\beta=0$, then we have the following:

Corollary 1. Assume that Assumptions 1-3 hold. For given any positive constant $\alpha$, if there exist four symmetric positive definite matrices $P_{i}(i=1,2,3,4)$, and four diagonal positive definite matrices $P_{5}=\operatorname{diag}\left(p_{51}, \ldots, p_{5 n}\right), P_{6}=\operatorname{diag}\left(p_{61}, \ldots, p_{6 n}\right), \wedge=\operatorname{diag}\left(\pi_{1}, \ldots, \pi_{n}\right)$ and $\Gamma=\operatorname{diag}\left(\xi_{1}, \ldots, \xi_{n}\right)$ such that (12), (13) $\left.\right|_{\beta=0}$, and (15) $\left.\right|_{\beta=0}$ hold, then the error system (8) is synchronized under the memory controller (7) with $\theta=1$ in finite time:

$$
T_{3}=\frac{\ln \left(1+\frac{\alpha V^{\frac{1-\mu}{2}}(0)}{2 k_{2}}\right)}{\frac{1-\mu}{2} \alpha} .
$$


Remark 3. This trivial case is discussed in Ref. [26]. In the study, the authors considered global finite-time observer problem.

In the following, several simplified version of Theorem 1 is derived for the case of $\alpha=\beta=0$.

Corollary 2. Assume that Assumptions 1-3 hold. If there exist four symmetric positive definite matrices $P_{i}(i=1,2,3,4)$, and four diagonal positive definite matrices $P_{5}=\operatorname{diag}\left(p_{51}, \ldots, p_{5 n}\right), P_{6}$ $=\operatorname{diag}\left(p_{61}, \ldots, p_{6 n}\right), \wedge=\operatorname{diag}\left(\pi_{1}, \ldots, \pi_{n}\right)$ and $\Gamma=\operatorname{diag}\left(\xi_{1}, \ldots, \xi_{n}\right)$ such that (12) $\left.\right|_{\alpha=0},\left.(13)\right|_{\beta=0}$, and (15) $\left.\right|_{\alpha=\beta=0}$ hold, then the error system (8) is synchronized under the periodically intermittent memory controller (7) in finite time:

$$
T_{4}=\frac{V^{\frac{1-\mu}{2}}(0)}{\frac{1-\mu}{2} k_{2} \theta}
$$

Let $\alpha=\beta=0$, and $\theta=1$, then Corollary 2 is derived as follows:

Corollary 3. Assume that Assumptions 1-3 hold. If there exist four symmetric positive definite matrices $P_{i}(i=1,2,3,4)$, and four diagonal positive definite matrices $P_{5}=\operatorname{diag}\left(p_{51}, \ldots, p_{5 n}\right), P_{6}$ $=\operatorname{diag}\left(p_{61}, \ldots, p_{6 n}\right), \wedge=\operatorname{diag}\left(\pi_{1}, \ldots, \pi_{n}\right)$ and $\Gamma=\operatorname{diag}\left(\xi_{1}, \ldots, \xi_{n}\right)$ such that $\left.(12)\right|_{\alpha=0}$ hold, then the error system (8) is synchronized under the memory controller (7) with $\theta=1$ in finite time:

$$
T_{5}=\frac{V^{\frac{1-\mu}{2}}(0)}{\frac{1-\mu}{2} k_{2}}
$$

Remark 4. Corollary 2 in this paper is the main result in Ref. [20]. This corollary is the simple case of Theorem 1. In this corollary, the differential of Lypunov function in the non-control width satisfied $\dot{V}(t)<0$ is not easily implemented. It is clear that this corollary has been investigated by previous Refs. [27]-[30], which is a continuous control problem.

Remark 5. In Refs. [19, 20], we have analysed that applying an appropriate continuous control (if the control rate $\theta=1$ ) in the systems for achieving the synchronization time is faster than the intermittent control (if the control rate $0<\theta<1$ ), that is, $T_{3} \leq T_{2}$ and $T_{5} \leq T_{4}$. On the other hand, it is easy to obtain that $T_{2} \leq T_{1}, T_{3} \leq T_{5}$ and $T_{2} \leq T_{4}$. Thus, the convergence time $T_{i}(i=1,2, \ldots, 5)$ satisfy: $T_{3} \leq T_{2} \leq T_{1}, T_{3} \leq T_{2} \leq T_{4}, T_{3} \leq T_{5} \leq T_{4}$. Besides, if $\theta=0.5$, the following inequality also holds: $T_{1} \leq T_{4}$, since the scalar constants satisfy: $\alpha>\beta$.

\section{Numerical example}

In this section, a numerical example is given to show the validity of the derived results.

Example 1. Consider two-nodes delayed neural network models (1) and (2) with the system state variables $x(t)=\left(x_{1}(t), x_{2}(t)\right)^{T}, y(t)=\left(y_{1}(t), y_{2}(t)\right)^{T}$, the external input $J(t)=(0,0)$, the discrete delay $\tau(t)=0.6|\sin (5 t)|$, the distributed delay $\sigma(t)=0.1|\cos (2 t)|$, and the system matrices

$$
C=\left[\begin{array}{cc}
1 & 0 \\
0 & 0.5
\end{array}\right], A=\left[\begin{array}{cc}
1.7 & -0.12 \\
-5.2 & 3.5
\end{array}\right], B=\left[\begin{array}{cc}
-1.7 & -0.12 \\
-0.25 & -2.4
\end{array}\right], D=\left[\begin{array}{ll}
-1.2 & -0.1 \\
-2.8 & -0.9
\end{array}\right] \text {. }
$$

Take the activation functions as $f(x)=\left[1 /\left(1+x_{1}^{2}\right), 1 /\left(1+x_{2}^{2}\right)\right]^{T}$. Fig. 1 shows the chaotic attractor (1) with a initial condition $x(t)=(-0.2,-0.3)^{T}, t \in[-0.6,0]$. Now, it is straightforward to 


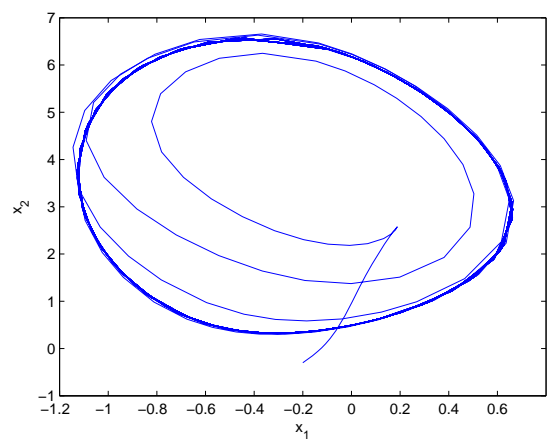

Fig. 1. Chaotic attractor of system (1).

check that all conditions in Theorem 1 hold. The systems (1) and (2) satisfies Assumptions 1 with $l_{1}^{-}=l_{2}^{-}=0$ and $l_{1}^{+}=l_{2}^{+}=1$, then we have

$$
\begin{aligned}
& L_{1}=\left[\begin{array}{ll}
0 & 0 \\
0 & 0
\end{array}\right], L_{2}=\left[\begin{array}{cc}
\frac{1}{2} & 0 \\
0 & \frac{1}{2}
\end{array}\right], L_{3}=\left[\begin{array}{ll}
1 & 0 \\
0 & 1
\end{array}\right], \\
& L_{4}=\left[\begin{array}{ll}
0 & 0 \\
0 & 0
\end{array}\right], L_{5}=\left[\begin{array}{ll}
0 & 0 \\
0 & 0
\end{array}\right], L_{6}=\left[\begin{array}{ll}
1 & 0 \\
0 & 1
\end{array}\right] .
\end{aligned}
$$

By using Matlab LMI Toolbox, and let $\mu=0.5$ and $\lambda=70$, it can be easily verified that the LMIs (12) and (13) are feasible and the solution can be obtained as follows:

$$
\begin{gathered}
P_{1}=\left[\begin{array}{cc}
15.9105 & 5.3733 \\
5.3733 & 14.7478
\end{array}\right], P_{2}=\left[\begin{array}{cc}
77.175 & -0.205 \\
-0.205 & 77.175
\end{array}\right], P_{3}=\left[\begin{array}{cc}
78.451 & 0.852 \\
0.852 & 78.451
\end{array}\right], \\
P_{4}=\left[\begin{array}{cc}
10.960 & 0.243 \\
0.243 & 10.960
\end{array}\right], P_{5}=\left[\begin{array}{cc}
360.017 & 0 \\
0 & 360.017
\end{array}\right], P_{6}=\left[\begin{array}{cc}
349.035 & 0 \\
0 & 349.035
\end{array}\right], \\
\Gamma=\left[\begin{array}{cc}
312.370 & 0 \\
0 & 313.257
\end{array}\right], \Lambda=\left[\begin{array}{cc}
534.270 & 0 \\
0 & 524.341
\end{array}\right],
\end{gathered}
$$

and $k_{1}=43.0464, k_{2}=1.0658, k_{3}=8.9867, \alpha=0.3240, \beta=1.0713$. From (14) and (15) in theorem 1 , we get $\theta<\frac{1.0713}{1.3953}=0.7678$. For numerical simulation, the initial conditions $x(0)=[-0.2,-0.3]^{T}, y(0)=[0.3,0.5]^{T}$, and the parameters $\theta=0.75, T=2$. Then, it follows from Theorem 1 that system (1) can finite-time synchronize with the desired system (2) under the periodically intermittent memory feedback (7). The simulation results are given in Figs. 2-4.

From the parameters of Figs. 2-4, by choosing an appropriate parameter $\alpha$, the convergence time of Fig. 3 is faster than that of Fig. 2; the convergence time of Fig. 4 is faster than that of Fig. 3 . This observation is elaborated in Remark 5. Therefore, the simulation results have confirmed the effectiveness of our proposed methods. 


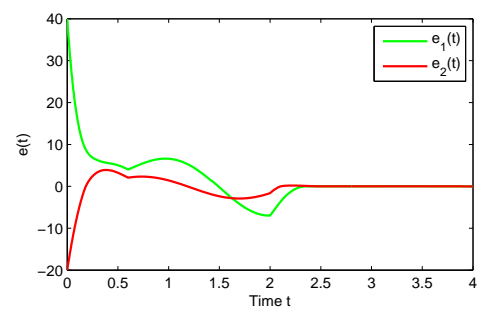

Fig. 2. Time response curve of neural networks with the periodically intermittent memory control (7) under the parameters $k_{1}=43.0464, k_{2}=1.0658, k_{3}=8.9867, \alpha=0.324, \beta=1.0713, \theta=$ $0.75, T=2, \mu=0.5$.

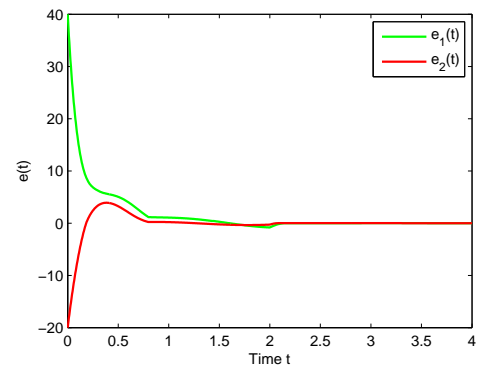

Fig. 3. Time response curve of neural networks with the periodically intermittent memory control (7) under the parameters $k_{1}=43.0464, k_{2}=1.0658, k_{3}=8.9867, \alpha=0.8, \beta=1.0713, \theta=$ $0.75, T=2, \mu=0.5$

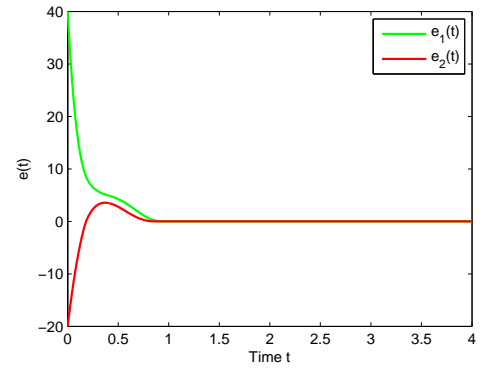

Fig. 4. Time response curve of neural networks with the periodically intermittent memory control (7) under the parameters $k_{1}=43.0464, k_{2}=1.0658, k_{3}=8.9867, \alpha=1.4, \beta=1.0713, \theta=$ $0.75, T=2, \mu=0.5$ 


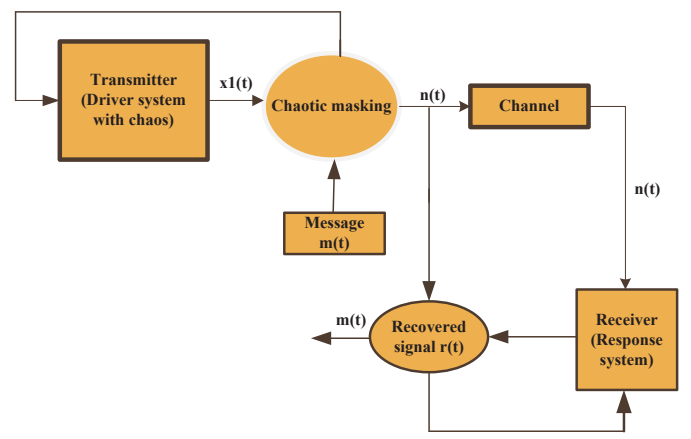

Fig. 5. Secure communication system based on finite-time synchronization

\section{Application to secure communication}

In secure communication, it is expected that only two entities communicate while the third entity is impossible to understand. For this purpose, encryption schemes are designed to avoid messages eavesdropped and intercepted. Chaos encryption is usually an effective way to improve safety of communication. The communication network can be regarded as chaotic neural networks, in which the sender is regarded as the drive system and the receiver as the response system. In this section, the memory feedback synchronization proposed in Theorem 1 is applied to make the receiver exactly follow the sender in finite time. The discrete and distributed time delays are complicated, which leads more difficulties of interpreting and unmasking the encrypted messages. In this application, an information signal $m(t)$ containing the transmitted messages can be masked with mixed time delays into the carrier $x(t)$, then the actual signal transmitted $n(t)$ is obtained for communication. Different strategies can be used to make the actual transmitted signal $n(t)$ as broadband as possible, so that its detection is tough via spectral techniques. In general, there exist three strategies in chaotic secure communication [40]. The first one is signal masking, where $n(t)=x(t)+\varepsilon m(t)$; the second is modulation, where $n(t)=x(t) m(t)$; the third is to combine the masking and modulation, such as $n(t)=x(t)[1+\varepsilon m(t)]$. For easy illustration, we only use the first masking strategy in this paper as an example. The finite-time synchronization can be applied to extract the message at the side of each receiver in the communication network. Unless synchronization is achieved with finite time, each receiver cannot accurately interpret messages transmitted. Fig. 5 shows the structure of presented communication system that consists a sender (or master) and a receiver (or slave), in which the transmitted signal is $n(t)=x(t)+\varepsilon m(t)$. By the proposed periodically intermittent memory feedback synchronization scheme, the message signal with the injected time delays (parameters of communication) is then derived in finite time at each receiving end. The sender and the receiver are designed as follows. The sender is designed as

$$
\begin{aligned}
\dot{x}_{1}(t)= & -0.8 x_{1}(t)+\sum_{j=1}^{2} a_{1 j} f_{j}\left(x_{j}(t)\right)+\sum_{j=1}^{2} b_{1 j} f\left(x_{j}(t-\tau(t))\right)+ \\
& \sum_{j=1}^{2} d_{1 j} \int_{t-\sigma(t)}^{t} f_{j}\left(x_{j}(s)\right) \mathrm{d} s+m_{1}(t),
\end{aligned}
$$




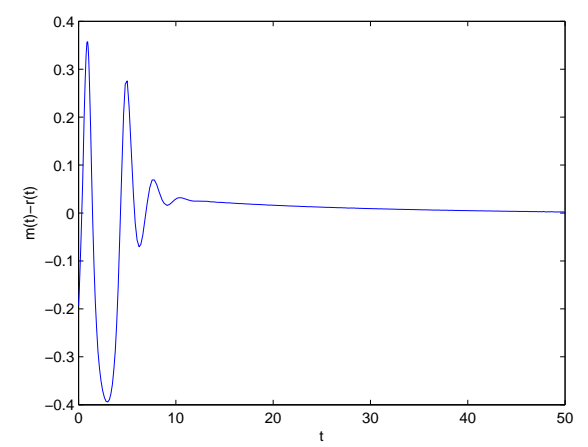

Fig. 6. Error between the transmitted signal $m(t)$ and the recovered signal $r(t)$

$$
\begin{aligned}
\dot{x}_{2}(t)= & -0.4 x_{2}(t)+\sum_{j=1}^{2} a_{2 j} f_{j}\left(x_{j}(t)\right)+\sum_{j=1}^{2} b_{2 j} f\left(x_{j}(t-\tau(t))\right)+ \\
& \sum_{j=1}^{2} e_{2 j} \int_{t-\sigma(t)}^{t} f_{j}\left(x_{j}(s)\right) \mathrm{d} s,
\end{aligned}
$$

where the system parameters are the same as the numerical example in Section IV. We assume that $m_{1}(t)=\varepsilon m(t)$ is the information message, and let $m_{2}(t)=0$. It should be denoted that the message signal must consume lower power, i.e., be weak in comparison with the chaotic carrier. Therefore, $\varepsilon=0.05$ is selected.

The receiver is designed as

$$
\begin{aligned}
\dot{y}_{1}(t)= & -0.8 y_{1}(t)+\sum_{j=1}^{2} a_{1 j} f_{j}\left(y_{j}(t)\right)+\sum_{j=1}^{2} b_{1 j} f\left(y_{j}(t-\tau(t))\right)+ \\
& \sum_{j=1}^{2} d_{1 j} \int_{t-\sigma(t)}^{t} f_{j}\left(y_{j}(s)\right) \mathrm{d} s+u_{1}(t)-y_{1}(t)+n_{1}(t), \\
\dot{y}_{2}(t)= & -0.4 x_{2}(t)+\sum_{j=1}^{2} a_{2 j} f_{j}\left(x_{j}(t)\right)+\sum_{j=1}^{2} b_{2 j} f\left(x_{j}(t-\tau(t))\right)+ \\
& \sum_{j=1}^{2} d_{2 j} \int_{t-\sigma(t)}^{t} f_{j}\left(x_{j}(s)\right) \mathrm{d} s+u_{2}(t)-y_{2}(t)+n_{2}(t),
\end{aligned}
$$

where $n_{1}(t)=n(t)=x_{1}(t)+\varepsilon m(t)$ is the transmitted signal, and for the sake of finite-time synchronization, we also assume $n_{2}(t)=x_{2}(t)$. The information message can be recovered by $r(t)=\varepsilon^{-1}\left[n(t)-y_{1}(t)\right]$. In the simulation, we take the same parameters and function as Example 1 in Section 4, and choose the information message as $m(t)=\sin (0.05)$. Fig. 6 depicts the error system between the chaotic neural networks (41)-(42) with transmitted signal $m(t)$ and the response systems (43)-(44) with message $r(t)$. From the simulation result, we can obtain that the signal can be exactly recovered by using the periodically intermittent memory feedback controller. The developed result shows the simulation perfectly. 


\section{Conclusion}

In this paper, the finite-time synchronization of a class of neural networks with discrete and distributed delays is newly studied. The periodical intermittent memory feedback control scheme is designed to derive the system achieving finite-time synchronization. Based on the finite-time stability theory and differential inequality, the finite-time synchronization criteria for such a system are obtained by using LMIs. The proposed condition (15) in Theorem 1 is required in the finite-time intermittent control. If this condition is ignored, the results of Theorem 1 and corollaries in this paper are not sufficient to be the finite-time intermittent control problem. Numerical simulations have proven that the proposed synchronization criteria are efficient for finite-time synchronization. The finite-time synchronization has been successfully applied to secure communication. In this application, the time delays make the message transmission safer and more accurate. Each receiver can exactly and effectively interpret the transmitted messages within a finite time.

\section{Acknowledgment}

The authors would like to thank the editor and the anonymous reviewers for their valuable comments and constructive suggestions.

This research is supported by National Natural Science Foundation of China (Grant No. 61273183, No. 61374028 and No. 61374085).

\section{References}

[1] L. M. Pecora, T. L. Carroll, Synchronization in chaotic systems, Phys. Rev. Lett., 64 (1990): 821-825.

[2] L. M. Pecora, T. L. Carroll, G. A. Johnson, J. F. Heagy, Fundamentals of synchronization in chaotic systems, concepts, and applications, Chaos 7, 520 (1997).

[3] G. Chen, X. Dong, From Chaos to Order: Methodologies, Perspectives, and Applications, World Scientific, Singapore, 1998.

[4] S. Baccaletti, J. Kurths, G. Osipov, D. L. Valladares, C. Zhou, The synchronization of chaotic systems, Phys. Rep., 366 (2002) 1-101.

[5] S. P. Gualberto, Complete synchronization of stictly different chaotic systems, Journal of Applied Mathematics, (2012) 964179 .

[6] X. Yang, J. Cao, Y. Long, W. Rui, Adaptive lag synchronization for competitive neural networks with mixed delays and uncertain hybrid perturbations, IEEE Trans. Neural Netw., 21(10) (2010) 1656-1667.

[7] G. Mahmoud, E. Mahmoud, Phase and antiphase synchronization of two identical hyperchaotic complex nonlinear systems, Nonlinear Dyn., 61 (2010) 141-152.

[8] W. Wang, H. Peng, L. Li, J. Xiao, Y. Yang, Finite-time function projective synchronization in complex multi-links networks with time-varying delay, Neural Processing Letters, 41 (2015) 71-88. 
[9] Y. Sun, W. Li, J. Ruan, Generalized outer synchronization between complex dynamical networks with time delay and noise perturbation, Commun Nonlinear Sci Numer Simulat, 18 (2013) 989-998.

[10] X. Yang, J. Cao, J. Lu, Stochastic synchronization of complex networks with nonidentical nodes via hybrid adaptive and impulsive control, IEEE Trans. Circuits Syst. I, 59 (2012) 371384.

[11] W. Wu, W. Zhou, T. Chen, Cluster synchronization of linearly coupled complex networks under pinning control, IEEE Trans. Circuits Syst. I, 56 (2009) 829-839.

[12] W. Zhang, Y. Tang, Q. Miao, J. Fang, Synchronization of stochastic dynamical networks under impulsive control with time delays, IEEE Trans. Neural Netw. Lear. Sys., 25(10) (2014) 1758-1768.

[13] M. Jiang, S. Wang, J. Mei, Y. Shen, Finite-time synchronization control of a class of memristor-based recurrent neural networks, Neural Networks, 63 (2015) 133-140.

[14] M. Jiang, J. Mei, J. Hu, New results on exponential synchronization of memristor-based chaotic neural networks, Neurocomputing, 156 (2015) 60-67.

[15] G. Zhang, Y. Shen, Exponential stabilization of memristor-based chaotic neural networks with time-Varying delays via intermittent control, IEEE Trans. Neural Netw. Learn. Syst., DOI: 10.1109/TNNLS.2014.2345125.

[16] J. Mei, M. Jiang, B. Wang, Q. Liu, W. Xu, T. Liao, Exponential p-synchronization of nonautonomous Cohen-Grossberg neural networks with reaction-diffusion terms via periodically intermittent control, Neural Processing Letters, 40 (2014) 103-126.

[17] C. Hu, J. Yu, H. Jiang, Z. Teng, Exponential synchronization of complex networks with finite distributed delays coupling, IEEE Trans. Neural Netw., 22 (2011) 1999-2010.

[18] X. Liu, T. Chen, Cluster synchronization in directed networks via intermittent pinning control, IEEE Trans. Neural Netw., 22(7) (2011) 1009-1020.

[19] J. Mei, M. Jiang, W. Xu, B. Wang, Finite-time synchronization control of complex dynamical networks with time delay, Commun Nonlinear Sci Numer Simulat, 18 (2013) 2462-2478.

[20] J. Mei, M. Jiang, X. Wang, J. Han, S. Wang, Finite-time synchronization of drive-response systems via periodically intermittent adaptive control, Journal of The Franklin Institute, 351 (2014) 2691-2710.

[21] X. Liu, T. Chen, Synchronization of nonlinear coupled networks via aperiodically intermittent pinning control, IEEE Trans. Neural Netw. Lear. Sys., 26(1) (2015) 113-126.

[22] J. Mei, M. Jiang, Z. Wu, X. Wang, Periodically intermittent controlling for finite-time synchronization of complex dynamical networks, Nonlinear Dyn., 79(1) (2014) 295-305.

[23] Y. Fan, H. Liu, J. Mei, Semi-global finite-time synchronization of complex dynamical networks via periodically intermittent control, Abstract and Applied Analysis, DOI: http://www.hindawi.com/journals/aaa/aip/381078/. 
[24] Z. Yan, W. Zhang, G. Zhang, Finite-time stability and stabilization of It stochastic system$s$ with Markovian switching: mode-dependent parameters approach, IEEE Transactions on Automatic Control, 60 (2015), 2428-2433.

[25] Z. Yan, G. Zhang, W. Zhang, Finite-time stability and stabilization of linear It stochastic systems with state and control dependent noise, Asian Journal of Control, 15 (2013), 270-281.

[26] Y. Shen, Y. Huang, Uniformly observable and globally lipschitzian nonlinear system admit global finite-time observers, IEEE Trans. Autom. Control, 54 (2009) 2621-2625.

[27] J. Mei, M. Jiang, B. Wang, B. Long, Finite-time parameter identification and adaptive synchronization between two chaotic neural networks, Journal of The Franklin Institute, 350 (2013) 1617-1633.

[28] J. Mei, M. Jiang, J. Wang, Finite-time structure identification and synchronization of driveresponse systems with uncertain parameter, Commun Nonlinear Sci Numer Simulat, 18 (2013) 999-1015.

[29] J. Yin, S. Khoo, Z. Man, X. Yu, Finite-time stability and instability of stochastic nonlinear systems, Automatica, 47 (2011) 2671-2677.

[30] X. Yang, J. Cao, Finite-time stochastic synchronization of complex networks, Appl. Math. Modeling, 34 (2010) 3631-3641.

[31] J. Shen, J. Cao, Finite-time synchronization of coupled neural networks via discontinuous controllers, Cogn Neurodyn, 5 (2011) 373385.

[32] W. Yu, Finite-time stabilization of three-dimensional chaotic systems based on CLF, Phys. Lett. A, 374 (2010) 3021-3024.

[33] T. Wang, S. Zhao, W. Zhou, W. Yu, Finite-time master-slave synchronization and parameter identification for uncertain Lurie systems, ISA Transactions, 53 (2014) 1184-1190.

[34] C. Hu, J. Yu, H. Jiang, Finite-time synchronization of delayed neural networks with CohenGrossberg type based on delayed feedback control, Neurocomputing, 143 (2014) 90-96.

[35] J. Huang, C. Li, T. Huang, X. He, Finite-time lag synchronization of delayed neural networks, Neurocomputing, 139 (2014) 145-149.

[36] W. Cui, J. Fang, W. Zhang, X. Wang, Finite-time cluster synchronisation of Markovian switching complex networks with stochastic perturbations, IET Control Theory and Applications, 8 (2013) 3041.

[37] Y. Liu, Z. Wang, X. Liu, Global exponential stability of generalized recurrent neural networks with discrete and distributed delays, Neural Networks, 19 (2006) 667-675.

[38] L. Wang, F. Xiao, Finite-time consensus problems for networks of dynamic agents, IEEE Trans. Autom. Control, 55 (2010) 950-955.

[39] B. Zhang, J. Lam, S. Xu, Stability analysis of distributed delay neural networks based on relaxed LyapunovKrasovskii functionals, IEEE Trans. Neural Netw. Lear. Sys., DOI: 10.1109/TNNLS.2014.2347290. 
[40] Y. Xia, Z. Yang, M. Han, Lag synchronization of unknown chaotic delayed Yang-Yang-type fuzzy neural networks with noise perturbation based on adaptive control and parameter identification, IEEE Trans. Neural Netw., 20 (2009) 1165-1180.

\section{Appendix}

Proof of Lemma 4: Take $M_{0}=V^{1-\eta}(0)+\frac{\alpha}{p_{1}}$ and $W(t)=V^{1-\eta}(t) \exp \left\{(1-\eta) p_{1} t\right\}$, where $t \geq 0$. Let $Q(t)=W(t)-M_{0}+\frac{\alpha}{p_{1}} \exp \left\{(1-\eta) p_{1} t\right\}$. It is easy to see that

$$
Q(t)=0, \quad \text { for } t=0 .
$$

In the following, we will prove that

$$
Q(t) \leq 0, \quad \text { for all } t \in[0, \theta T) .
$$

If this is not true, then there exists a $t_{1} \in[0, \theta T)$ such that

$$
\begin{array}{ll}
Q\left(t_{1}\right)=0, & \dot{Q}\left(t_{1}\right)>0, \\
Q(t) \leq 0, & 0 \leq t<t_{1} .
\end{array}
$$

Using Eqs. (45), (47) and (48), we obtain

$$
\begin{aligned}
\dot{Q}\left(t_{1}\right)= & (1-\eta) V^{-\eta}\left(t_{1}\right) \dot{V}\left(t_{1}\right) \exp \left\{(1-\eta) p_{1} t_{1}\right\}+p_{1}(1-\eta) V^{1-\eta}\left(t_{1}\right) \exp \left\{(1-\eta) p_{1} t_{1}\right\}+ \\
& \alpha(1-\eta) \exp \left\{(1-\eta) p_{1} t_{1}\right\} \\
\leq & (1-\eta) V^{-\eta}\left(t_{1}\right)\left(-\alpha V^{\eta}\left(t_{1}\right)-p_{1} V\left(t_{1}\right)\right) \exp \left\{(1-\eta) p_{1} t_{1}\right\}+ \\
& p_{1}(1-\eta) V^{1-\eta}\left(t_{1}\right) \exp \left\{(1-\eta) p_{1} t_{1}\right\}+\alpha(1-\eta) \exp \left\{(1-\eta) p_{1} t_{1}\right\} \\
= & -\alpha(1-\eta) \exp \left\{(1-\eta) p_{1} t_{1}\right\}-p_{1}(1-\eta) V^{1-\eta}\left(t_{1}\right) \exp \left\{(1-\eta) p_{1} t_{1}\right\} \\
& +p_{1}(1-\eta) V^{1-\eta}\left(t_{1}\right) \exp \left\{(1-\eta) p_{1} t_{1}\right\}+\alpha(1-\eta) \exp \left\{(1-\eta) p_{1} t_{1}\right\} \\
= & 0 .
\end{aligned}
$$

This contradicts the second inequality in (47), and so (46) holds.

Let $W_{1}(t)=V^{1-\eta}(t) \exp \left\{(1-\eta) p_{1} t\right\} \exp \left\{-(1-\eta) p_{1}(t-\theta T)\right\} \exp \left\{-(1-\eta) p_{2}(t-\theta T)\right\}$, and $H(t)=W_{1}(t)-M_{0}+\frac{\alpha}{p_{1}} \exp \left\{(1-\eta) p_{1} t\right\} \exp \left\{-(1-\eta) p_{1}(t-\theta T)\right\} \exp \left\{-(1-\eta) p_{2}(t-\theta T)\right\}, t \geq \theta T$. Next, we prove that for $t \in[\theta T, T)$,

$$
H(t) \leq 0, \quad \text { for all } t \in[\theta T, T) .
$$

Otherwise, there exists a $t_{2} \in[\theta T, T)$ such that

$$
\begin{array}{ll}
H\left(t_{2}\right)=0, & \dot{H}\left(t_{2}\right)>0, \\
H(t) \leq 0, & \theta T \leq t<t_{2} .
\end{array}
$$


According to Eqs. (51) and (52), we have

$$
\begin{aligned}
\dot{H}\left(t_{2}\right)= & \dot{W}_{1}\left(t_{2}\right)+\frac{\alpha}{p_{1}}(1-\eta) p_{1} \exp \left\{(1-\eta) p_{1} t_{2}\right\}\left[\operatorname { e x p } \left\{-(1-\eta) p_{1}\left(t_{2}-\theta T\right)\right.\right. \\
& \exp \left\{-(1-\eta) p_{2}\left(t_{2}-\theta T\right)\right]+\frac{\alpha}{p_{1}} \exp \left\{(1-\eta) p_{1} t_{2}\right\}\left[-(1-\eta)\left(p_{1}+p_{2}\right)\right. \\
& \left.\exp \left\{-(1-\eta)\left(p_{1}+p_{2}\right)\left(t_{2}-\theta T\right)\right\}\right] \\
= & (1-\eta) V^{-\eta}\left(t_{2}\right) \dot{V}\left(t_{2}\right) \exp \left\{(1-\eta) p_{1} t_{2}\right\} \exp \left\{-(1-\eta)\left(p_{1}+p_{2}\right)\left(t_{2}-\theta T\right)\right\}+ \\
& V^{1-\eta}\left(t_{2}\right)\left[\exp \left\{(1-\eta) p_{1} t_{2}\right\} \exp \left\{-(1-\eta) p_{1}\left(t_{2}-\theta T\right)\right\} \exp \{-(1-\eta)\right. \\
& \left.\left.p_{2}\left(t_{2}-\theta T\right)\right\}\right]^{\prime}+\frac{\alpha}{p_{1}}(1-\eta) p_{1} \exp \left\{(1-\eta) p_{1} t_{2}\right\} \exp \left\{-(1-\eta)\left(p_{1}+p_{2}\right)\right. \\
& \left.\left(t_{2}-\theta T\right)\right\}-\frac{\alpha}{p_{1}}(1-\eta)\left(p_{1}+p_{2}\right) \exp \left\{(1-\eta) p_{1} t_{2}\right\} \exp \{-(1-\eta) \\
& \left.\left(p_{1}+p_{2}\right)\left(t_{2}-\theta T\right)\right\} \\
\leq & p_{2}(1-\eta) V^{1-\eta}\left(t_{2}\right) \exp \left\{(1-\eta) p_{1} t_{2}\right\} \exp \left\{-(1-\eta)\left(p_{1}+p_{2}\right)\left(t_{2}-\theta T\right)\right\} \\
& +V^{1-\eta}\left(t_{2}\right)\left[( 1 - \eta ) p _ { 1 } \operatorname { e x p } \{ ( 1 - \eta ) p _ { 1 } t _ { 2 } \} \operatorname { e x p } \left\{-(1-\eta)\left(p_{1}+p_{2}\right)\right.\right. \\
& \left(t_{2}-\theta T\right)-(1-\eta)\left(p_{1}+p_{2}\right) \exp \left\{(1-\eta) p_{1} t_{2}\right\} \exp \left\{-(1-\eta)\left(p_{1}+p_{2}\right)\right. \\
& \left.\left.\left(t_{2}-\theta T\right)\right\}\right]+\frac{\alpha}{p_{1}}(1-\eta) p_{1} \exp \left\{(1-\eta) p_{1} t_{2}\right\} \exp \left\{-(1-\eta)\left(p_{1}+p_{2}\right)\left(t_{2}-\right.\right. \\
& \theta T)\}-\frac{\alpha}{p_{1}}(1-\eta)\left(p_{1}+p_{2}\right) \exp \left\{(1-\eta) p_{1} t_{2}\right\} \exp \left\{-(1-\eta)\left(p_{1}+p_{2}\right)\left(t_{2}-\theta T\right)\right\} \\
= & p_{2}(1-\eta) V^{1-\eta}\left(t_{2}\right) \exp \left\{(1-\eta) p_{1} t_{2}\right\} \exp \left\{-(1-\eta)\left(p_{1}+p_{2}\right)\left(t_{2}-\theta T\right)\right\}- \\
& p_{2}(1-\eta) V^{1-\eta}\left(t_{2}\right) \exp \left\{(1-\eta) p_{1} t_{2}\right\} \exp \left\{-(1-\eta)\left(p_{1}+p_{2}\right)\left(t_{2}-\theta T\right)\right\}-\frac{\alpha}{p_{1}} \\
& p_{2} \exp \left\{(1-\eta) p_{1} t_{2}\right\} \exp \left\{-(1-\eta)\left(p_{1}+p_{2}\right)\left(t_{2}-\theta T\right)\right\} \\
< & 0,
\end{aligned}
$$

which contradicts the second inequality in (51). Hence (50) holds. From (50), it is easy to see that

$$
\begin{aligned}
W(t) \leq & \exp \left\{(1-\eta)\left(p_{1}+p_{2}\right)(t-\theta T)\right\}\left[M_{0}-\frac{\alpha}{p_{1}} \exp \left\{(1-\eta) p_{1} t\right\} \exp \{-(1-\right. \\
& \left.\left.\eta)\left(p_{1}+p_{2}\right)(t-\theta T)\right\}\right] .
\end{aligned}
$$

Consequently, for $t \in[\theta T, T)$, we have

$$
\begin{aligned}
W(t)< & \exp \left\{(1-\eta)\left(p_{1}+p_{2}\right)(t-\theta T)\right\}\left[M_{0}-\right. \\
& \left.\frac{\alpha}{p_{1}} \exp \left\{(1-\eta) p_{1} t\right\} \exp \left\{-(1-\eta)\left(p_{1}+p_{2}\right)(t-\theta T)\right\}\right] \\
\leq & \exp \left\{(1-\eta)\left(p_{1}+p_{2}\right)(1-\theta) T\right\}\left[M_{0}-\right. \\
& \left.\frac{\alpha}{p_{1}} \exp \left\{(1-\eta) p_{1} t\right\} \exp \left\{-(1-\eta)\left(p_{1}+p_{2}\right)(1-\theta) T\right\}\right] .
\end{aligned}
$$


On the other hand, it follows from Eqs. (45) and (46) that for $t \in[0, \theta T)$,

$$
\begin{aligned}
W(t) \leq & M_{0}-\frac{\alpha}{p_{1}} \exp \left\{(1-\eta) p_{1} t\right\} \\
\leq & M_{0}-\frac{\alpha}{p_{1}} \exp \left\{(1-\eta) p_{1} t\right\} \exp \left\{-(1-\eta)\left(p_{1}+p_{2}\right)(1-\theta) T\right\} \\
\leq & \exp \left\{(1-\eta)\left(p_{1}+p_{2}\right)(1-\theta) T\right\}\left[M_{0}-\frac{\alpha}{p_{1}} \exp \left\{(1-\eta) p_{1} t\right\} \exp \left\{-(1-\eta)\left(p_{1}\right.\right.\right. \\
& \left.\left.\left.+p_{2}\right)(1-\theta) T\right\}\right] .
\end{aligned}
$$

So

$$
\begin{aligned}
W(t)< & \exp \left\{(1-\eta)\left(p_{1}+p_{2}\right)(1-\theta) T\right\}\left[M_{0}-\right. \\
& \left.\frac{\alpha}{p_{1}} \exp \left\{(1-\eta) p_{1} t\right\} \exp \left\{-(1-\eta)\left(p_{1}+p_{2}\right)(1-\theta) T\right\}\right],
\end{aligned}
$$

for all $t \in[0, T)$.

Similarly, we can proof that for $t \in[T,(1+\theta) T)$,

$$
\begin{aligned}
W(t)< & \exp \left\{(1-\eta)\left(p_{1}+p_{2}\right)(1-\theta) T\right\}\left[M_{0}-\right. \\
& \left.\frac{\alpha}{p_{1}} \exp \left\{(1-\eta) p_{1} t\right\} \exp \left\{-(1-\eta)\left(p_{1}+p_{2}\right)(1-\theta) T\right\}\right],
\end{aligned}
$$

and for $t \in[(1+\theta) T, 2 T)$,

$$
\begin{aligned}
W(t)< & \exp \left\{(1-\eta)\left(p_{1}+p_{2}\right)(1-\theta) T\right\} \exp \left\{(1-\eta)\left(p_{1}+p_{2}\right)(t-\theta T-T)\right\} \\
& {\left[M_{0}-\frac{\alpha}{p_{1}} \exp \left\{(1-\eta) p_{1} t\right\} \exp \left\{-(1-\eta)\left(p_{1}+p_{2}\right)(1-\theta) T\right\} \exp \left\{-(1-\eta)\left(p_{1}+\right.\right.\right.} \\
& \left.\left.\left.p_{2}\right)(t-\theta T-T)\right\}\right] \\
= & \exp \left\{(1-\eta)\left(p_{1}+p_{2}\right)(t-2 \theta T)\right\}\left[M_{0}-\frac{\alpha}{p_{1}} \exp \left\{(1-\eta) p_{1} t\right\} \exp \{-(1-\eta)\right. \\
& \left.\left.\left(p_{1}+p_{2}\right)(t-2 \theta T)\right\}\right]
\end{aligned}
$$

By induction, for any integers $m$, we can derive the following estimation of $W(t)$ for any $m$. For $m T \leq t<(m+\theta) T$,

$$
\begin{aligned}
W(t)< & \exp \left\{(1-\eta)\left(p_{1}+p_{2}\right)(1-\theta) m T\right\}\left[M_{0}-\frac{\alpha}{p_{1}} \exp \left\{(1-\eta) p_{1} t\right\}\right. \\
& \left.\exp \left\{-(1-\eta)\left(p_{1}+p_{2}\right)(1-\theta) m T\right\}\right]
\end{aligned}
$$

and for $(m+\theta) T \leq t<(m+1) T$,

$$
\begin{aligned}
W(t)< & \exp \left\{(1-\eta)\left(p_{1}+p_{2}\right)(t-(m+1) \theta T)\right\}\left[M_{0}-\frac{\alpha}{p_{1}}\right. \\
& \left.\exp \left\{(1-\eta) p_{1} t\right\} \exp \left\{-(1-\eta)\left(p_{1}+p_{2}\right)(t-(m+1) \theta T)\right\}\right] .
\end{aligned}
$$


Since for any $t \geq 0$, there exists a nonnegative integer $k$, such that $k T \leq t<(k+1) T$, we can deduce the following estimation of $W(t)$ for any $t$ by Eqs. (55) and (56).

For $k T \leq t<(k+\theta) T$,

$$
\begin{aligned}
W(t)< & \exp \left\{(1-\eta)\left(p_{1}+p_{2}\right)(1-\theta) k T\right\}\left[M_{0}-\frac{\alpha}{p_{1}}\right. \\
& \left.\exp \left\{(1-\eta) p_{1} t\right\} \exp \left\{-(1-\eta)\left(p_{1}+p_{2}\right)(1-\theta) k T\right\}\right] \\
\leq & \exp \left\{(1-\eta)\left(p_{1}+p_{2}\right)(1-\theta) t\right\}\left[M_{0}-\frac{\alpha}{p_{1}}\right. \\
& \left.\exp \left\{(1-\eta) p_{1} t\right\} \exp \left\{-(1-\eta)\left(p_{1}+p_{2}\right)(1-\theta) t\right\}\right]
\end{aligned}
$$

and for $(k+\theta) T \leq t<(k+1) T$,

$$
\begin{aligned}
W(t)< & \exp \left\{(1-\eta)\left(p_{1}+p_{2}\right)(t-(k+1) \theta T)\right\}\left[M_{0}-\right. \\
& \left.\frac{\alpha}{p_{1}} \exp \left\{(1-\eta) p_{1} t\right\} \exp \left\{-(1-\eta)\left(p_{1}+p_{2}\right)(t-(k+1) \theta T)\right\}\right] \\
\leq & \left.\exp \left\{(1-\eta)\left(p_{1}+p_{2}\right)(1-\theta) t\right)\right\}\left[M_{0}-\right. \\
& \left.\frac{\alpha}{p_{1}} \exp \left\{(1-\eta) p_{1} t\right\} \exp \left\{-(1-\eta)\left(p_{1}+p_{2}\right)(1-\theta) t\right\}\right] .
\end{aligned}
$$

Together with the definition of $W(t)$, we obtain

$$
\begin{aligned}
\left.V^{1-\eta}(t) \exp \left\{(1-\eta) p_{1} t\right)\right\} \leq & \exp \left\{(1-\eta)\left(p_{1}+p_{2}\right)(1-\theta) t\right\}\left[V^{1-\eta}(0)+\frac{\alpha}{p_{1}}\right. \\
& \left.-\frac{\alpha}{p_{1}} \exp \left\{(1-\eta) p_{1} \theta t\right\} \exp \left\{-(1-\eta) p_{2}(1-\theta) t\right\}\right] \\
= & \exp \left\{(1-\eta)\left(p_{1}+p_{2}\right)(1-\theta) t\right\}\left[V^{1-\eta}(0)-\frac{\alpha}{p_{1}}\right. \\
& \left.\left(\exp \left\{(1-\eta) p_{1} \theta t\right\} \exp \left\{-(1-\eta) p_{2}(1-\theta) t\right\}-1\right)\right] .
\end{aligned}
$$

It implies the conclusion and the proof is completed. 